\title{
INADEQUACIES OF CHISHOLM'S DEFINITIONS OF THE EVIDENT
}

\author{
MARCELO DASCAL \\ LYDIA AMIR \\ Tel-Aviv University
}

R. Chisholm proposed in the last fifteen years at least three different definitions of what it is for a proposition to be evident, namely in the first edition of Theory of Knowledge (1966), in the second edition of the same book (1977) and, more recently, in a 1979 paper. The changes in the definition are part of his constant effort to ammend and improve his analysis, in the light of criticism. They are not, therefore, arbitrary, but rather guided by certain criteria of adequacy. For example, one condition for the adequacy of any definition of the evident is set forth by the claim that whatever is known is evident (Chisholm, 1966: 20). If so, care must be taken in defining the evident so as not to let the definition reduce too much the range of what is known. Trying to face this danger, however, may lead to the opposite danger, namely that of a too permissive definition. We believe that two of the definitions proposed by Chisholm suffer from these opposite defects: one of them is too restrictive and the other, too permissive. The remaining definition is immune to such criticism, but conflicts with other requirements put forward by Chisholm himself. In this paper we try to substantiate these claims, by considering in detail the definitions proposed and by trying to find out whether the changes introduced, especially in the third definition, do indeed produce the hoped for improve. ments.

Let us begin by comparing Chisholm's various definitions of the evident. In the first edition of Theory of Knowledge, the evident is defined as follows: 
(1) $h$ is evident for $S=\operatorname{Df}$ (a) $h$ is beyond reasonable doubt for $S$ and (b) there is no proposition $i$ such that it is more reasonable for $S$ to believe $i$ than it is for him to believe $h$ (1966: 22).

At this time, the evident occupies the highest position amongst the five basic terms of epistemic appraisal. But in the second edition of the book, a new epistemic term, the 'certain', is defined exactly as was the evident in the first edition, and the highest epistemic praise is now reserved for this new term. The evident occupies now an intermediate position, between the 'certain' and that which is 'beyond reasonable doubt'. It is in fact defined by means of these terms:

(2) $h$ is evident for $S=\mathrm{Df}$ (a) $h$ is beyond reasonable doubt for $S$ and (b) for every $i$, if accepting $i$ is more reasonable for $S$ than accepting $h$, then $i$ is certain for $S$ (1977: 12).

Later on, still unsatisfied, Chisholm redefines the evident, now using a notion which he did not make use of so far, namely the notion of 'confirmation':

(3) $h$ is evident for $S$ at $t=\operatorname{Df}$ (a) $h$ is beyond reasonable doubt for $S$ at $t$ and (b) no conjunction of propositions that are acceptable for $S$ at $t$ tends to confirm not- $h$ (1979: 122).

Consider definition (1). Clearly it is too restrictive. For, according to it, only propositions that are not exceeded in reasonableness by any other proposition are evident. Hence, since being evident is a necessary condition for being known (1966: 20), it turns out that, on definition (1), the range of what is known is quite restricted. So much is fairly clear, and it may indeed be the reason for Chisholm's later modifications of the definition. 
It is perhaps less obvious that definition (3) jumps to the other extreme, namely excessive permissiveness. This is what we purport to show presently. For that purpose, let us recall what are Chisholm's expectations from this new definition: 'an adequate definition of the evident - he says - should enable us to say, of that which is evident, that it is more than merely beyond reasonable doubt and yet that it need not be certain' (1979: 122). We submit that the evident, as defined in (3), does not fulfill the first of these requirements, namely that of being 'more than merely beyond reasonable doubt'. In other words, we claim that every proposition that is beyond reasonable doubt for $S$ is, by definition (3), evident for $S$. In order to show this, let us take any proposition $p$ that is beyond reasonable doubt for $S$, and let us check whether it satisfies definition (3). The first condition ( $p$ is beyond reasonable doubt for $S$ ) is satisfied ex hypothesi. As for the second condition, let us assume that $p$ does not satisfy it, i.e. that there is a conjunction $e$ of acceptable propositions (for $S$ ), which tends to confirm not-p. If we apply to this last statement the definition of ' $e$ tends to confirm $h$ ' $\left(={ }^{\prime} e\right.$ is necessarily such that, for every subject $S$, if $e$ is beyond reasonable doubt for $S$ and if everything that is beyond reasonable doubt for $S$ is logically implied by $e$, then $h$ has some presumption in its favor'), we obtain: not- $p$ has some presumption in its favor (i.e. accepting not- $p$ is more reasonable than accepting $p$ ). We have thus a proposition $p$, which is beyond reasonable doubt (by the hypothesis), whereas its negation has some presumption in its favor. This is by itself a fairly uncomfortable situation. Since someone might still argue that, albeit unusual, this situation is not altogether impossible, let us show that it involves a formal contradiction. There are two ways of doing so. The first, and most immediate one, is simply to recall that every proposition that is beyond reasonable doubt has some presumption in its favor (Chisholm, 1977:8). The contradictory statement is, then, that both $p$ and not- $p$ have some presumption in their favor. The other way is to use one of the basic principles governing the use of the un- 
defined expression 'more reasonable than'. According to this principle, if withholding $p$ is not more reasonable than accepting $p$, then accepting $p$ is more reasonable than accepting not-p (Chisholm, 1977: 13). Schematically:

$$
\text { (4) } W(p) \leqslant A(p) \supset A(p)>A(\sim p)
$$

(where ' $W(p)$ ' stands for ' $(S)$ withholds $p$ ', 'A(p)' stands for ' $(S)$ accepts $p$ ', ' $>$ ' stands for 'is more reasonable than', and ' $\leqslant$ ' for its negation). Since $p$ is beyond reasonable doubt, we can say that accepting $p$ is more reasonable than withholding it, that is:

$$
\text { (5) } A(p)>W(p) \text {. }
$$

And since not-p has some presumption in its favor, we know that accepting not- $p$ is more reasonable than accepting $p$, that is:

(6) $A(\sim p)>A(p)$.

But, from (4) and (5) we obtain:

(7) $A(p)>A(\sim p)$

and, from (6) and (7), we can derive the contradiction:

(8) $(A(p)>A(\sim p)) \&(A(\sim p)>A(p))$.

This contradiction, as well as the former, was derived, you will recall, on the assumption that $p$ does not satisfy the second condition of definition (3). Hence, this assumption must be false. That is to say, every proposition that is beyond reasonable doubt must satisfy definition (3) and is, consequently, evident. The definition thus reduces the evident to what is beyond reasonable doubt. It does not let the evident occupy the intermediate position Chisholm wants it to occupy. In this sense, definition (3) can be said to be too permissive. 
To sum up: when the evident is identified with what is beyond reasonable doubt (definition (3)), its definition is excessively permissive; when the evident is identified with the certain (definition (1)), its definition is too restrictive. Definition (2), as opposed to the other two, is tailored precisely to assign to the evident the intermediate position required. By this criterion, therefore, it cannot be neither too permissive nor too restrictive. If so, why should Chisholm abandon it?

Chisholm wrote the 1979 paper in order to correct his views on the directly evident. His earlier theory on this subject was based on the (mistaken) general presupposition to the effect that 'the vehicles of the directly evident are primarily certain ' $I$ '-propositions - my ' $I$ '-propositions being the propositions that I would express in English by using the first person and your ' $I$ '-propositions being the ones that you would express in English by using the first person. The theory...presupposes that the propositional knowledge that each person has is based upon that person's 'I'-propositions' (1979: 116). But, given Chisholm's current skepticism as to whether there are any ' $I$ '-propositions (as opposed to mere ' $I$ '-sentences), the theory requires, for him, ammendment. The gist of the paper is thus to propose a new characterization of the self-presenting (which is the basic concept out of which the directly evident is construed), without appealing to 'I'-propositions. Yet, at the same time, and for no apparent reason, Chisholm also modifies his definition of the evident. The latter, it will be recalled, is not defined in terms of the self-presenting, so that a change in the definition of this concept does not require necessarily any change in that of the evident. One is thus justified in wondering why did Chisholm replace the apparently satisfactory definition (2) - which he had put forward only two years earlier - by a definition which is manifestly over-permissive.

This puzzling move might eventually be explained by pointing out that, in spite of its apparent adequacy, definition (2) 
is in fact deficient in some respect. Thus, Wasserman has pointed out that this definition yields two unacceptable results: (a) "Knowledge comes in only two degrees of reasonableness' (i.e. the evident and the certain); and (b) 'nothing we know about the external world is more reasonable than anything else we know about it' (because all we know about the external world is noncertain and evident). Wasserman gives five arguments showing how these implications of Chisholm's definition arise. These arguments - especially the first (p. 43) - show indeed that, contrary to Chisholm's assertion (1977: 12), definition (2) violates his own condition of 'uniqueness', namely the requirement that the evident, like the certain, should not be capable of degrees. A variant of Wasserman's first argument is worth presenting, because it shows a different possible source for the generation of degrees within the evident. Our argument relies on one of Chisholm's assertions in the second edition, and on a reasonable assumption. These are:

(9) A proposition can be both evident and false (1977: 15); and

(10) It is more reasonable to accept a proposition that can be known, than to accept a proposition that cannot be known.

('More reasonable than' has here the intuitive sense assigned to it by Chisholm who, it will be recalled, does not define this locution.) Now, we may know or not know a given proposition, but clearly, if a proposition is false, we cannot possibly know it. Therefore if (10) is granted, then to accept a proposition $p$ which is true and evident is more reasonable than to accept a proposition $q$ which is false and evident. ${ }^{1}$

1 Since this is all we need in the argument that follows, a less general, and more plausible, formulation of (10) should perhaps be the one used here. Something like: All other things being equal, it is more reasonable for $S$ to accept a proposition which is true and evident (for him), than to accept a proposition that is false, and evident (for him). 
Since both types of proposition do in fact exist (by (9)), one must conclude that, assuming (10), there are certain other kinds of evident propositions which are more reasonable than other evident propositions. In order to ensure that these are indeed degrees of reasonableness within the domain of the evident, we have to show that these more reasonable propositions are not necessarily certain. For that purpose, consider the following example. Let $p$ be the evident and true proposition that there is a cat in the roof, and let $q$ be the evident but false proposition that the sun is moving around us in the sky. According to (10), accepting $p$ is more reasonable than accepting $q$. But $p$ does not belong to the realm of the certain since it is possible to find a proposition $i$ such that $i$ is more reasonable than $p$. Such an $i$ might be, for example, the proposition that I take there to be a cat on the roof. This proposition is, on Chisholm's theory, self-presenting, whereas the proposition that there is a cat on the roof is only evident (1977: 64). Now, there are two possibilities: either $i$ is merely evident, or it is certain. If the former is the case, then there are at least three degrees within the realm of the evident, exemplified by $p, q$ and $i$; but even if $i$ is certain, there remain still two degrees within the evident, exemplified by $p$ and $q$. (According to the second edition, a self-presenting proposition like $i$ is evident, while according to Person and Object - p. 179, it is certain.) In any case, the evident as defined by (2) has been shown to have degrees, being thus unable to comply with the condition of uniqueness.

Although the 1979 paper may contribute to comply with this condition, due to the changes it introduces in the notion of the self-presenting, definition (3) by itself does not seem to prevent the generation of degrees within the evident: it may even make them proliferate, through its reduction of the evident to that which is beyond reasonable doubt. The change introduced by Chisholm in his definition of the evident appears thus to be even more gratuituous, since it not only 
does not contribute to compensate for the one defect we were able to detect in (2), but also creates new problems of its own.

\section{REFERENCES}

Chisholm, R. (1966), Theory of Knowledge, lst ed., Englewood-Cliffs, Prentice-Hall.

(1976), Person and Object, London, George Allen \& Unwin.

(1977), Theory of Knowledge, 2d. ed,, Englewood Cliffs, Prentice. Hall.

(1979), "The Directly Evident", in George S. Pappas (ed.), Justififation and Knowledge, 115-127.

Wasserman, W. (1980), "Chisholm's definition of the evident", Analysis: 42-44. 